\title{
Correction to: Relationally equal treatment of equals and affine combinations of values for TU games
}

\section{Koji Yokote ${ }^{1} \cdot$ Takumi Kongo $^{2} \cdot$ Yukihiko Funaki $^{1}$}

Published online: 19 July 2019

๑) Springer-Verlag GmbH Germany, part of Springer Nature 2019

\section{Correction to: Social Choice and Welfare https://doi.org/10.1007/s00355-019-01180-y}

We are grateful to Zhengxing Zou for pointing out an insufficiency in the definition of an element of our new basis for the space of TU games, $w_{T}$, in Sect. 5.1. With keeping the definition of $\left(N, w_{T}\right) \in \Gamma$ as it is for $|T| \neq \frac{n}{2}$, we modify it by

$$
w_{T}=u_{T}-\sum_{\ell \in N \backslash T} u_{N \backslash \ell}
$$

only for $|T|=\frac{n}{2}$.

According to this modification, the following changes are required.

Proof of Lemma 1 This immediately follows from the fact that $|T| \geq 3$ and $|N| \geq 3$.

Proof of Lemma 2 For $|T| \neq \frac{n}{2}$, the proof is as it is. For $|T|=\frac{n}{2}$, if $i \in T, w_{T}(N \backslash i)=$ $0-0=0$, and if $i \notin T, w_{T}(N \backslash i)=u_{T}(N \backslash i)-u_{N \backslash i}(N \backslash i)=1-1=0$.

Publisher's Note Springer Nature remains neutral with regard to jurisdictional claims in published maps and institutional affiliations.

The original article can be found online at https://doi.org/10.1007/s00355-019-01180-y.

$凶$ Takumi Kongo

kongo@adm.fukuoka-u.ac.jp

Koji Yokote

sidehand@toki.waseda.jp

Yukihiko Funaki

funaki@waseda.jp

1 Faculty of Political Science and Economics, Waseda University, 1-6-1, Nishi-Waseda, Shinjuku-ku, Tokyo 169-8050, Japan

2 Faculty of Economics, Fukuoka University, 8-19-1 Nanakuma, Jonan-ku, Fukuoka 814-0180, Japan 